\title{
A PROPERTY OF THE FROBENIUS MAP OF A POLYNOMIAL RING
}

\author{
GENNADY LYUBEZNIK, WENLIANG ZHANG AND YI ZHANG
}

\begin{abstract}
Let $R=k\left[x_{1}, \ldots, x_{n}\right]$ be a ring of polynomials in a finite number of variables over a perfect field $k$ of characteristic $p>0$ and let $F: R \rightarrow R$ be the Frobenius map of $R$, i.e. $F(r)=r^{p}$. We explicitly describe an $R$-module isomorphism $\operatorname{Hom}_{R}\left(F_{*}(M), N\right) \cong$ $\operatorname{Hom}_{R}\left(M, F^{*}(N)\right)$ for all $R$-modules $M$ and $N$. Some recent and potential applications are discussed.
\end{abstract}

\section{INTRODUCTION}

The main result of this paper is Theorem 3.3 which is a type of adjointness property for the Frobenius map of a polynomial ring over a perfect field. The interest in this fairly elementary result comes from its striking recent applications (see [5, Sections 5 and 6] and [6]) and also from the fact that despite extensive inquiries we have not been able to find it in the published literature.

Especially interesting is the application in 6] where a striking new result on local cohomology modules in characteristic $p>0$ is deduced from Theorem 3.3. There is no doubt that the same result is true in characteristic 0 , but the only currently known (to us) proof is in characteristic $p>0$, based on Theorem 3.3. This has motivated what promises to be a very interesting search for some new technique to extend the result in [6] to characteristic 0 .

We believe the results of this paper hold a potential for further applications and we discuss some of them in the last section.

\section{Preliminaries}

Let $R$ be a regular (but not necessarily local) UFD, let $R_{s}$ and $R_{t}$ be two copies of $R$ (the subscripts stand for source and target) and let $F: R_{s} \rightarrow R_{t}$ be a finite ring homomorphism. Let

$$
F_{*}: R_{t}-\bmod \rightarrow R_{s}-\bmod
$$

The first and third authors gratefully acknowledge NSF support through grants DMS-0202176 and DMS-0701127. The second author is supported in part by a 2009 Spring/Summer Research Fellowship from Department of Mathematics, University of Michigan. 
be the restriction of scalars functor (i.e. $F_{*}(M)$ for every $R_{t}$-module $M$ is the additive group of $M$ regarded as an $R_{s}$-module via $F$ ) and let

$$
F^{!}, F^{*}: R_{s}-\bmod \rightarrow R_{t}-\bmod
$$

be the functors defined by $F^{!}(N)=\operatorname{Hom}_{R_{s}}\left(R_{t}, N\right)$ and $F^{*}(N)=R_{t} \otimes_{R_{s}} N$ for every $R_{s}$-module $N$.

$F^{*}(N)$ and $F^{!}(N)$, for an $R_{s}$-module $N$, have a structure of $R_{s}$-module via the natural $R_{s}$-action on $R_{t}$, while $F_{*}(M)$, for an $R_{t}$-module $M$, retains its old $R_{t}$-module structure (from before the restriction of scalars). Thus $F^{*}(N), F^{!}(N)$, and $F_{*}(M)$ are both $R_{s^{-}}$and $R_{t^{-} \text {-modules. }}$.

It is well-known that $F^{*}$ is left-adjoint to $F_{*}$, i.e. there is an isomorphism of $R_{t}$-modules

$$
\begin{aligned}
\operatorname{Hom}_{R_{t}}\left(F^{*}(N), M\right) & \cong \operatorname{Hom}_{R_{s}}\left(N, F_{*}(M)\right) \\
f & \mapsto(n \mapsto f(1 \otimes n)) \\
(r \otimes n \mapsto r f(n)) & \leftarrow f
\end{aligned}
$$

which is functorial in $M$ and $N$ (see, for example, [2, II.5, p.110]). This holds without any restrictions on $R$.

The main result of this paper is based on a different type of adjointness (see 8 below) which is certainly not well-known (we could not find a reference). Unlike (1) it is not quite canonical but depends on a choice of a certain isomorphism $\phi$ (described in (6) below). And it requires the conditions we imposed on $R$, namely, a regular UFD.

Since $R_{t}$, being regular, is locally Cohen-Macaulay, it follows from the Auslander-Buchsbaum theorem that the projective dimension of $R_{t}$ as $R_{s^{-}}$ module is zero, i.e. $R_{t}$ is projective, hence locally free, as an $R_{s}$-module. It is a standard fact that in this case $F^{!}$is right adjoint to $F_{*},[2$, Ch. 3, Exercise 6.10] i.e. for every $R_{t}$-module $M$ and every $R_{s}$-module $N$ there is an $R_{t}$-module isomorphism

$$
\begin{aligned}
\operatorname{Hom}_{R_{s}}\left(F_{*}(M), N\right) & \cong \operatorname{Hom}_{R_{t}}\left(M, F^{!}(N)\right) \\
(m \mapsto \mathfrak{f}(1)) & \leftarrow f
\end{aligned}
$$

where $\mathfrak{f}=f(m): R_{t} \rightarrow N$. This isomorphism is functorial both in $M$ and in $N$.

Consider the $R_{t}$-module $H \stackrel{\text { def }}{=} \operatorname{Hom}_{R_{s}}\left(R_{t}, R_{s}\right)$. Since $R_{t}$ is a locally free $R_{s}$-module of finite rank, it is a standard fact [2, Ch 2, Exercise 5.1(b)] that there is an isomorphism of functors

$$
F^{!} \cong H \otimes_{R_{s}}-
$$

i.e. for every $R_{s}$-module $N$ there is an $R_{t}$-module isomorphism

$$
\begin{aligned}
H \otimes_{R_{s}} N & \cong \operatorname{Hom}_{R_{s}}\left(R_{t}, N\right) \\
h \otimes n & \mapsto(r \mapsto h(r) n)
\end{aligned}
$$


and this isomorphism is functorial in $N$. Replacing $F^{!}$by $H \otimes_{R_{s}}-$ in (2) produces an $R_{t}$-module isomorphism

$$
\operatorname{Hom}_{R_{s}}\left(F_{*}(M), N\right) \cong \operatorname{Hom}_{R_{t}}\left(M, H \otimes_{R_{s}} N\right) .
$$

which is functorial in $M$ and $N$.

It follows from [3, Kor. 5.14] that locally $H$ is the canonical module of $R_{t}$. In particular, the rank of $H$ as $R_{t}$-module is 1 and therefore $H$ is $R_{t}$-module isomorphic to some ideal $I$ of $R_{t}$. According to [3, Kor. 6.13] the quotient $R_{t} / I$ is locally Gorenstein of dimension $\operatorname{dim} R_{t}-1$, hence $I$ has pure height 1. Since $R_{t}$ is a UFD, $I$ is principal, i.e. there is an $R_{t}$-module isomorphism

$$
\phi: R_{t} \rightarrow H
$$

Hence $\phi$ induces an isomorphism of functors

$$
R_{t} \otimes_{R_{s}}-\stackrel{\phi \otimes i \mathrm{id}}{\cong} H \otimes_{R_{s}}-
$$

Replacing $H \otimes_{R_{s}}-$ by $F^{*}=R_{t} \otimes_{R_{s}}-$ in (5) via (7) produces an $R_{t}$-module isomorphism

$$
\operatorname{Hom}_{R_{s}}\left(F_{*}(M), N\right) \cong \operatorname{Hom}_{R_{t}}\left(M, F^{*}(N)\right)
$$

which is functorial in $M$ and $N$.

While the isomorphisms (2), (3), (44) and (5) are canonical, the isomorphisms (6), (7) and (86) depend on a choice of $\phi$. Every $R_{t}$-module isomorphism $\phi^{\prime}: R_{t} \rightarrow H$ is obtained from a fixed $\phi$ by multiplication by an invertible element of $R_{t}$, i.e. $\phi^{\prime}=c \cdot \phi$ where $c \in R_{t}$ is invertible. Therefore the isomorphisms (7) and (8) are defined up to multiplication by an invertible element of $R_{t}$.

Since the element $1 \in R_{t}$ generates the $R_{s}$-submodule $F\left(R_{s}\right)$ of $R_{t}$ and does not belong to $\mathfrak{m} R_{t}$ for any maximal ideal $\mathfrak{m}$ of $R_{s}$, the $R_{s}$-module $R_{t} / F\left(R_{s}\right)$ is projective. Hence applying the functor $\operatorname{Hom}_{R_{s}}\left(-, R_{s}\right)$ to the injective map $F: R_{s} \rightarrow R_{t}$ produces a surjection $H \rightarrow \operatorname{Hom}_{R_{s}}\left(R_{s}, R_{s}\right)$. Composing it with the standard $R_{s}$-module isomorphism $\operatorname{Hom}_{R_{s}}\left(R_{s}, R_{s}\right) \stackrel{\psi \mapsto \psi(1)}{\longrightarrow}$ $R_{s}$ produces an $R_{s}$-module surjection $H \rightarrow R_{s}$. Composing this latter map with the isomorphism $\phi$ from (6) produces an $R_{s}$-module surjection

$$
\psi: R_{t} \rightarrow R_{s}
$$

If $N$ is an $R_{s}$-module, applying $-\otimes_{R_{s}} N$ to $\psi$ produces an $R_{s}$-module surjection

$$
\psi_{N}: R_{t} \otimes_{R_{s}} N \rightarrow N .
$$

It is not hard to check that the isomorphism (8) sends $g \in \operatorname{Hom}_{R_{t}}\left(M, F^{*}(N)\right)$ to $\psi_{N} \circ g \in \operatorname{Hom}_{R_{s}}\left(F_{*}(M), N\right)$. 


\section{The Main Result}

For the rest of this paper $R$ is a ring of polynomials in a finite number of variables over a perfect field $k$ of characteristic $p>0$ and $F: R_{s} \rightarrow R_{t}$ is the standard Frobenius map, i.e. $F(r)=r^{p}$. The main result of this paper (Theorem 3.3) is an explicit description of the isomorphism (8) in terms of polynomial generators of $R$. The recent applications [5, 6] crucially depend on this explicit description. We keep the notation of the preceding section.

Let $x_{1}, \ldots, x_{n}$ be some polynomial generators of $R$ over the field $k$, i.e. $R=k\left[x_{1}, \ldots, x_{n}\right]$. We denote the multi-index $i_{1}, \ldots, i_{n}$ by $\bar{i}$. Since $k$ is

perfect, $R_{t}$ is a free $R_{s}$-module on the $p^{n}$ monomials $e_{i} \stackrel{\text { def }}{=} x_{1}^{i_{1}} \cdots x_{n}^{i_{n}}$ where $0 \leq i_{j}<p$ for every $j$. If $i_{j}<p-1$, then $x_{j} e_{\bar{i}}=e_{\bar{i}^{\prime}}$ where $\bar{i}^{\prime}$ is the multiindex $i_{1}, \ldots, i_{j-1}, i_{j}+1, i_{j+1}, \ldots, i_{n}$. If $i_{j}=p-1$, then $x_{j} e_{\bar{i}}=x_{j}^{p} e_{\bar{i}^{\prime}}$ where $\bar{i}^{\prime}$ is the multi-index $i_{1}, \ldots, i_{j-1}, 0, i_{j+1}, \ldots, i_{n}$.

Let $\left\{f_{\bar{i}} \in H \mid 0 \leq i_{j}<p\right.$ for every $\left.j\right\}$ be the dual basis of $H$, i.e. $f_{\bar{i}}\left(e_{\bar{i}^{\prime}}\right)=1$ if $\bar{i}=\bar{i}^{\prime}$ and $f_{\bar{i}}\left(e_{\bar{i}^{\prime}}\right)=0$ otherwise. If $i_{j}>0$, then $x_{j} f_{\bar{i}}=f_{\bar{i}^{\prime}}$ where $\bar{i}^{\prime}$ is the multi-index $i_{1}, \ldots, i_{j-1}, i_{j}-1, i_{j+1}, \ldots, i_{n}$. If $i_{j}=0$, then $x_{j} f_{\bar{i}}=x_{j}^{p} f_{\bar{i}^{\prime}}$ where $\bar{i}^{\prime}$ is the multi-index $i_{1}, \ldots, i_{j-1}, p-1, i_{j+1}, \ldots, i_{n}$.

Denote the multi-index $p-1, \ldots, p-1$ by $\overline{p-1}$ and let $\overline{p-1}-\bar{i}$ be the multi-index $p-1-i_{1}, \ldots, p-1-i_{n}$.

Proposition 3.1. (cf. [1, Remark 3.11]) The $R_{s}$-linear isomorphism $\phi$ : $R_{t} \rightarrow H$ that sends $e_{\bar{i}}$ to $f_{\overline{p-1}-\bar{i}}$ is $R_{t}$-linear.

Proof. All we have to show is that $\phi\left(x_{j} e_{\bar{i}}\right)=x_{j} \phi\left(e_{\bar{i}}\right)$ for all indices $j$ and multi-indices $\bar{i}$. This is straightforward from the definition of $\phi$ and the above description of the action of $x_{j}$ on $e_{\bar{i}}$ and $f_{\bar{i}}$.

Clearly, $F^{*}(N)=R_{t} \otimes_{R_{s}} N=\oplus_{\bar{i}}\left(e_{\bar{i}} \otimes_{R_{s}} N\right)$, as $R_{s}$-modules. Thus every $R_{s}$-linear map $g: M \rightarrow F^{*}(N)$ has the form $g=\oplus_{\bar{i}}\left(e_{\bar{i}} \otimes_{R_{s}} g_{\bar{i}}\right)$ where $g_{\bar{i}}: M \rightarrow N$ are $R_{s}$-linear maps (i.e. $g_{\bar{i}}: F_{*}(M) \rightarrow N$ because $M$ with its $R_{s}$-module structure is $\left.F_{*}(M)\right)$.

Lemma 3.2. An $R_{s}$-linear map $g: M \rightarrow F^{*}(N)$ as above is $R_{t}$-linear if and only if $g_{\bar{i}}(-)=g_{\overline{p-1}}\left(e_{\overline{p-1}-\bar{i}}(-)\right)$ for every $\bar{i}$ (here $e_{\overline{p-1}-\bar{i}} \in R_{t}$ acts on $(-) \in F_{*}(M)$ via the $R_{t}$-module structure on $F_{*}(M)$ ).

Proof. Assume $g$ is $R_{t}$-linear. Then $g$ commutes with multiplication by every element of $R_{t}$ and in particular with multiplication by $e_{\overline{p-1}-\bar{i}}$. That is $g\left(e_{\overline{p-1}-\bar{i}}(-)\right)=e \overline{p-1}-\bar{i} g(-)$. Since $e_{\overline{p-1}-\bar{i}} e_{\bar{i}}=e \overline{p-1}$, the $\overline{p-1}$-component of $e_{\overline{p-1}-\bar{i}} g(-)$ is $e_{\overline{p-1}} \otimes_{R_{s}} g_{\bar{i}}(-)$ while the $\overline{p-1}$-component of $g\left(e_{\overline{p-1}-\bar{i}}(-)\right)$ is $e_{\overline{p-1}} \otimes_{R_{s}} g_{\overline{p-1}}\left(e_{\overline{p-1}-\bar{i}}(-)\right)$. Since the two $\overline{p-1}$-components are equal, $g_{\bar{i}}(-)=$ $g_{\overline{p-1}}(e \overline{p-1}-\bar{i}(-))$.

Conversely, assume $g_{\bar{i}}(-)=g_{\overline{p-1}}\left(e_{\overline{p-1}-\bar{i}}(-)\right)$ for every $\bar{i}$. To show that $g$ is $R_{t}$-linear all one has to show is that $g$ commutes with the action of every $x_{j} \in R_{t}$, i.e. the $\bar{i}$-components of $g\left(x_{j}(-)\right)$ and $x_{j} g(-)$ are the same for all $\bar{i}$. If $i_{j}>0$, then the $\bar{i}$-component of $x_{j} g(-)$ is $e_{\bar{i}} \otimes_{R_{s}} g_{\bar{i}^{\prime}}(-)$ where $\bar{i}^{\prime}$ is 
the index $i_{1}, \ldots, i_{j-1}, i_{j}-1, i_{j+1}, \ldots, i_{n}$ while the $\bar{i}$-component of $g\left(x_{j}(-)\right)$ is $e_{\bar{i}} \otimes_{R_{s}} g_{\bar{i}}\left(x_{j}(-)\right)$. But the fact that $g_{\bar{i}}(-)=g_{\overline{p-1}}(e \overline{p-1}-\bar{i}(-))$ for every $\bar{i}$ implies $g_{\bar{i}^{\prime}}(-)=g_{\bar{i}}\left(x_{j}(-)\right)$.

If $i_{j}=0$, then the $\bar{i}$-component of $x_{j} g(-)$ is $e_{\bar{i}^{\prime \prime}} \otimes_{R_{s}}\left({ }_{s} x_{j} g_{\bar{i}^{\prime \prime}}(-)\right)$ where ${ }_{s} x_{j}$ denotes the element of $R_{s}$ corresponding to $x_{j} \in R_{t}$ (i.e. $F\left({ }_{s} x_{j}\right)=x_{j}^{p}$ ) and $\bar{i}^{\prime \prime}$ is the index $i_{1}, \ldots, i_{j-1}, p-1, i_{j+1}, \ldots, i_{n}$ while the $\bar{i}$-component of $g\left(x_{j}(-)\right)$ is $g_{\bar{i}}\left(x_{j}(-)\right)$. But the fact that $g_{\bar{i}}(-)=g_{\overline{p-1}}\left(e_{\overline{p-1}-\bar{i}}(-)\right)$ for every $\bar{i}$ implies ${ }_{s} x_{j} g_{\bar{i}^{\prime \prime}}(-)=g_{\bar{i}}\left(x_{j}(-)\right)$.

Finally we are ready for the main result of the paper which is the following explicit description of the isomorphism (8) for the Frobenius map.

Theorem 3.3. For every $R_{t}$-module $M$ and every $R_{s}$-module $N$ there is an $R_{t}$-linear isomorphism

$$
\begin{aligned}
\operatorname{Hom}_{R_{s}}\left(F_{*}(M), N\right) & \cong \operatorname{Hom}_{R_{t}}\left(M, F^{*}(N)\right) \\
g_{\overline{p-1}}(-) & \leftarrow\left(g=\oplus_{\bar{i}}\left(e_{\bar{i}} \otimes_{R_{s}} g_{\bar{i}}(-)\right)\right) \\
g & \mapsto \oplus_{\bar{i}}\left(e_{\bar{i}} \otimes_{R_{s}} g\left(e_{\overline{p-1}-\bar{i}}(-)\right)\right) .
\end{aligned}
$$

Proof. As is pointed out at the end of the preceding section, the isomorphism (8) sends $g \in \operatorname{Hom}_{R_{t}}\left(M, F^{*}(N)\right)$ to $\psi_{N} \circ g \in \operatorname{Hom}_{R}\left(F_{*}(M), N\right)$. It is straightforward to check that with $\phi$ as in Proposition 3.1 the map $\psi$ of (9) sends $e_{\overline{p-1}}$ to 1 . This implies that $\psi_{N} \circ g=g_{\overline{p-1}}$ and finishes the proof that formula (11) produces the isomorphism of (8) in one direction. The fact that the other direction of this isomorphism is according to formula (12) has essentially been proven in Lemma 3.2.

\section{Potential Applications}

The notion of $F$-finite modules was introduced in [4]. An $F$-finite module is determined by a generating morphism, i.e. an $R$-module homomorphism $\beta: M \rightarrow F^{*}(M)$ where $M$ is a finite $R$-module. For simplicity assume the $R$-module $M$ has finite length, i.e. the dimension of $M$ as a vector space over $k$, which we denote by $d$, is finite. Then the dimension of $F^{*}(M)$ as a vector space over $k$ equals $p^{n} \cdot d$. The number $p^{n}$ can be huge even for quite modest values of $p$ and $n$. Thus the target of $\beta$ may be a huge-dimensional vector space even if $d, p$ and $n$ are fairly small. But the $R$-module $F_{*}(M)$ has dimension $d$ as a $k$-vector space, hence the map $\tilde{\beta}: F_{*}(M) \rightarrow M$ that corresponds to $\beta$ under the isomorphism of Theorem 3.3, is a map between two $d$-dimensional vector spaces. Huge-dimensional vector spaces do not appear! This should make the map $\tilde{\beta}$ easier to manage computationally than the map $\beta$. Of course the isomorphism of Theorem 3.3 means that many properties of $\beta$ could be detected in $\tilde{\beta}$; for example, $\beta$ is the zero map if and only if $\tilde{\beta}$ is. Therein lies the potential for using Theorem 3.3 to make computations more manageable. 
The functors $\operatorname{Ext}_{R}^{i}(-, R)$ commute with both $F^{*}$ and $F_{*}$. More precisely, for every finitely generated $R_{t}$-module $M$ and every finitely generated $R_{s^{-}}$ module $N$ there exist functorial $R_{t}$-module isomorphisms

$$
\begin{aligned}
& \kappa_{i}: \operatorname{Ext}_{R_{t}}^{i}\left(F^{*}(N), R_{t}\right) \cong F^{*}\left(\operatorname{Ext}_{R_{s}}^{i}\left(N, R_{s}\right)\right) \\
& \lambda_{i}: F_{*}\left(\operatorname{Ext}_{R_{t}}^{i}\left(M, R_{t}\right)\right) \cong \operatorname{Ext}_{R_{s}}^{i}\left(F_{*}(M), R_{s}\right) .
\end{aligned}
$$

Indeed, for $i=0, M=R_{t}$ and $N=R_{s}$ a straightforward composition of the $R_{t}$-module isomorphism $R_{t} \otimes_{R_{s}} R_{s} \stackrel{r^{\prime} \otimes r \mapsto r^{\prime} r^{p}}{\longrightarrow} R_{t}$ and the standard $R$ module isomorphisms $\operatorname{Hom}_{R}(R, R) \cong R$ for $R=R_{t}, R_{s}$ produce $\kappa_{0}$ while an additional $R_{t}$-module isomorphism $\operatorname{Hom}_{R_{s}}\left(R_{t}, R_{s}\right)=H \cong R_{t}$ produces $\lambda_{0}$. This implies that if - stands for a complex of finite free $R$-modules, then $\operatorname{Hom}_{R}(-, R)$ commutes with $F^{*}$ and $F_{*}$. Taking now finite free resolutions of $M$ and $N$ in the categories of $R_{t^{-}}$and $R_{s}$-modules respectively and considering that $F^{*}$ and $F_{*}$, being exact, commute with the operation of taking the (co)homology of complexes, we get $\kappa_{i}$ and $\lambda_{i}$ for every $i$.

It is straightforward to check that there is a commutative diagram

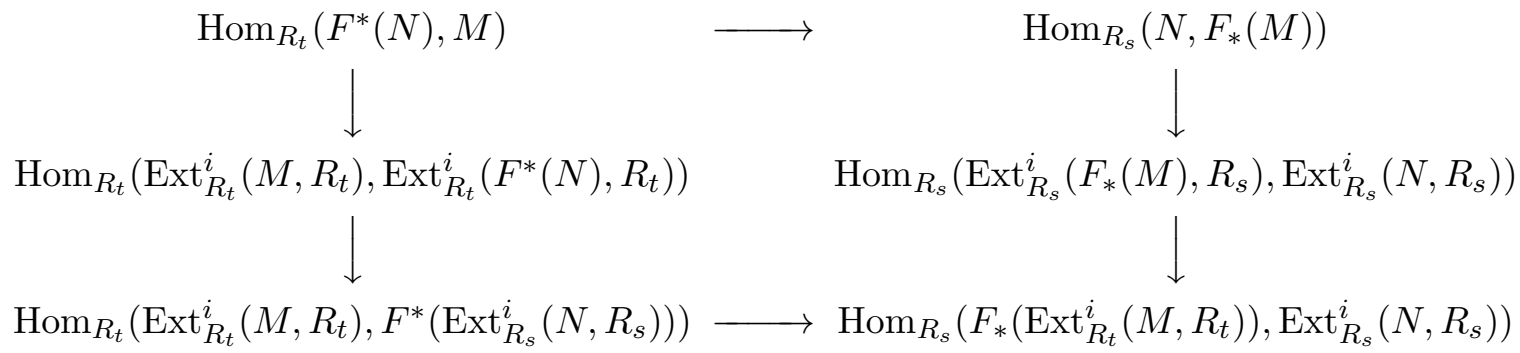

where the top horizontal map is the isomorphism (11), the bottom horizontal map is the isomorphism of Theorem 3.3. the bottom vertical maps are induced by $\kappa_{i}$ and $\lambda_{i}$ and, finally, the top vertical maps are defined by sending every $f \in \operatorname{Hom}\left(L, L^{\prime}\right)$ to the map $\operatorname{Ext}^{i}\left(L^{\prime}, R\right) \rightarrow \operatorname{Ext}^{i}(L, R)$ functorially induced by $f$.

In other words, if a pair of maps $F^{*}(N) \rightarrow M$ and $N \rightarrow F_{*}(M)$ correspond to each other under (11), then the induced maps $\operatorname{Ext}_{R_{t}}^{i}\left(M, R_{t}\right) \rightarrow$ $F^{*}\left(\operatorname{Ext}_{R_{s}}^{i}\left(N, R_{s}\right)\right)$ and $F_{*}\left(\operatorname{Ext}_{R_{t}}^{i}\left(M, R_{t}\right)\right) \rightarrow \operatorname{Ext}_{R_{s}}^{i}\left(N, R_{s}\right)$ correspond to each other under isomorphism of Theorem 3.3 .

Of course there is a similar diagram with the isomorphism of Theorem 3.3 in the top row; we are not going to use it in the rest of the paper.

An important example of $F$-finite modules are local cohomology modules $H_{I}^{i}(R)$ of $R$ with support in an ideal $I \subset R$. A generating morphism of $H_{I}^{i}(R)$ is the composition

$$
f: \operatorname{Ext}_{R}^{i}(R / I, R) \rightarrow \operatorname{Ext}_{R}^{i}\left(F^{*}(R / I), R\right) \stackrel{\kappa_{i}}{\cong} F^{*}\left(\operatorname{Ext}_{R}^{i}(R / I, R)\right)
$$

where the first map is induced by the isomorphism $F^{*}(R / I) \stackrel{r^{\prime} \otimes \bar{r} \mapsto r^{\prime} \bar{r}^{p}}{\cong} R / I^{[p]}$ followed by the natural surjecton $R / I^{[p]} \rightarrow R / I$. The following proposition 
holds the potential for simplifying computations involving local cohomology modules.

Proposition 4.1. Let $I \subset R$ be an ideal and let the composition

$$
f: \operatorname{Ext}_{R}^{i}(R / I, R) \rightarrow \operatorname{Ext}_{R}^{i}\left(F^{*}(R / I), R\right) \stackrel{\kappa_{i}}{\cong} F^{*}\left(\operatorname{Ext}_{R}^{i}(R / I, R)\right)
$$

be as above. The map that corresponds to $f$ under the isomorphism of Theorem 3.3 is the composition

$$
g: F_{*}\left(\operatorname{Ext}_{R}^{i}(R / I, R)\right) \stackrel{\lambda_{i}}{\cong} \operatorname{Ext}_{R}^{i}\left(F_{*}(R / I), R\right) \rightarrow \operatorname{Ext}_{R}^{i}(R / I, R)
$$

where the second map in the composition is nothing but the map induced on $\operatorname{Ext}_{R}^{i}(-, R)$ by the natural Frobenius map $R / I \stackrel{r \mapsto r^{p}}{\cong} F_{*}(R / I)$.

Proof. This is immediate from the above commutative diagram considering that the maps $F^{*}(R / I) \stackrel{r^{\prime} \otimes r \mapsto r^{\prime} r^{p}}{\longrightarrow} R / I$ and $R / I \stackrel{r \rightarrow r^{p}}{\rightarrow} F_{*}(R / I)$ correspond to each other under the isomorphism (11).

In addition to potential use in computation, the material of this section has already been used in a proof of a theoretical result [5, Section 5].

\section{REFERENCES}

[1] M. Blickle, K Schwede, S. Takagi and W. Zhang, Discreteness and Rationality of F-jumping Numbers on Singular Varieties, preprint, 2009, arXiv:0906.4679

[2] R. Hartshorne, Algebraic Geometry, Graduate Texts in Mathematics, 52, Springer 1977.

[3] J. Herzog and E. Kunst, Der Kanonische Modul eines Cohen-Macaulay-Rings, Lecture Notes in Mathematics 238, Springer 1971.

[4] G. Lyubeznik, F-modules: Applications to Local Cohomology and D-modules in Characteristic $p>0$, J. reine angew. Math., 491 (1997) $65-130$.

[5] W. Zhang, Lyubeznik Numbers of Projective Schemes, preprint, 2010.

[6] Y. Zhang, A Property Of Local Cohomology Modules Of Polynomial Rings, preprint, 2010.

Dept. of Mathematics, University of Minnesota, Minneapolis, MN 55455

E-mail address: gennady@math.umn.edu

Dept. of Mathematics, University of Michigan, Ann Arbor, Mi 48109.

E-mail address: wlzhang@umich.edu

Dept. of Mathematics, University of Minnesota, Minneapolis, MN 55455

E-mail address: zhang397@math.umn.edu 